\title{
Month Imputed
}

National Cancer Institute

\section{Source}

National Cancer Institute. Month Imputed. NCI Thesaurus. Code C154418.

An imputed date that includes the month of observation. 